\title{
Investigation on Silver Ion Release from Wound Dressings In Vitro and In Vivo
}

\author{
Liu $C^{*, 2}$, Shi $\mathrm{Y}^{1,2}$, Fan $\mathrm{C}^{1,2}$, Qu Z $\mathrm{Z}^{3}$, Liu $\mathrm{L}^{1,2}$, Hou $\mathrm{L}^{1,2}$, Wang $\mathrm{W}^{1,2}$, and Wang $\mathrm{X}^{1,2}$
}

${ }^{1}$ Shandong Quality Inspection Center for Medical Devices, Jinan, 250101, China

${ }^{2}$ Shandong Key Laboratory of Biological Evaluation for Medical Devices, Jinan, 250101, China

${ }^{3}$ Key Laboratory of Cardiovascular Remodeling and Function Research, Chinese Ministry of Education and Chinese Ministry of Public Health, Jinan, 250021, China

*Corresponding author: Liu C, Shandong Quality Inspection Center for Medical Devices, Jinan, 250101, China, Shandong Key Laboratory of Biological Evaluation for Medical Devices, NO.15166 Century Avenue, Jinan H-T Industrial Development Zone, Shandong Province 250101, P.R.China Fax:+86531-82682901, Tel: +86531-82682901, E-mail: liuchenghu510@163.com

Citation: Liu C, Shi Y, Fan C, Qu Z, Liu L, et al. (2017) Investigation on Silver Ion Release from Wound Dressings In Vitro and In Vivo. J Mater Sci Nanotechnol 5(2): 205

Received Date: October 23, 2017 Accepted Date: December 04, 2017 Published Date: December 11, 2017

\begin{abstract}
Introduction: In recent years, silver ion based dressings have been widely developed and approved in the market. Meanwhile, methods for selecting appropriate standard models to investigate the silver ion release in vitro and in vivo remains unknown with regard to the safety assessment.

Methods and Results: In this study, we have identified that silver ion release in eagle minimum essential mediums(MEM) with $10 \%$ fetal calf serum had shown better release kinetics in comparison to other vehicles including simulated body fluid (SBF), $0.9 \%$ physiological saline, and 5\% glucose. Further, both results of irritation tests in rabbits and antimicrobial efficacy determination further confirmed these scenarios in vitro. In addition, the results from in vivo tests indicated that rat models could be used as an appropriate model to simulate the silver ion release, just as intended in clinical practice.

Conclusion: Overall, our studies have demonstrated that MEM with $10 \%$ fetal calf serum and in vivo rat models can be used to determine the silver ion release from wound dressings in vitro and in vivo, respectively.

Keywords: Silver ion based dressing; Atomic Absorption Spectrometer (AAS); Irritation test; Antimicrobial efficacy; Rat model; Graphite Furnace(GF)
\end{abstract}

\section{Introduction}

Silver ion based wound dressings have been widely used to prevent infection during clinical application for abroad range of antimicrobial efficiency [1,2]. To date, it is well known that the efficacy of silver ion based dressings is basically dependent on the kinetics of silver ion release, which mainly depends upon the total silver content, application environment of dressings, and substrate materials where the silver ions were loaded [3,4]. Currently, silver ion release can be considered as the main reason for the potential toxicological risk and biocompatibility of these silver-based medical devices [5]. Further, some reports have demonstrated that silver ion release might lead to local silver deposition, delayed wound healing, and silver-induced renal toxicity [6,7].

So far, silver ion release in vitro has been fully illustrated by using different kinds of testing vehicles to simulate the scenarios of clinical application, along with the dressing characterization and release kinetics in vitro [8-10]. However, there are still some controversial issues regarding the selection of appropriate vehicles for silver ion release, since no specific standards have been developed. Some studies have already shown that media without serum proteins are more suitable for silver ion release, which maximizes its function due to the lack of potential interference between silver particles and proteins [11]. Others argue that chloride ions in the culture medium should be the real concerns for silver ion release, since they can bind to the silver ions released from the dressings [12,13].

In this study, based on the previous understanding of silver ion release, we used MEM with $10 \%$ fetal calf serum to further elucidate the kinetics of silver ion release in vitro. Furthermore, we compared the total silver ion release in this MEM cell medium with different sorts of comparable fluids, as well as using rat wound models to determine the silver ion release in vivo. To our knowledge, this investigation could provide useful information for silver ion release standardization, and contribute to the design 
and development of new and improved silver based dressings for better clinical practice.

\section{Materials and Methods}

\section{Materials}

Legally marketed highly absorbent dressings based on silver zirconium phosphate foam and comparator non-silver dressings were obtained from commercial sources. The silver nitrate (CAS Number, 7761-88-8) was purchased from Sigma Company. 0.9\% sodium chloride, simulated body fluid (SBF), and $5 \%$ glucose were prepared by the given formulary. For SBF ( $\mathrm{pH} 7.40$ ), the ion concentration (mM): $\mathrm{Na}^{+} 142.0 ; \mathrm{K}^{+} 5.0 ; \mathrm{Mg}^{2+} 1.5 ; \mathrm{Ca}^{2+} 2.5 ; \mathrm{Cl}^{-147.8} ; \mathrm{HCO}_{3}^{-4} .2 ; \mathrm{HPO}_{4}^{2-1} 1.0 ; \mathrm{SO}_{4}^{2-} 0.5$, and $\mathrm{MEM}$ cell cultures were purchased from Hangzhou Sijiqing Company, China.

\section{Animals}

Adult male Sprague-Dawley rats $(240 \mathrm{~g}-300 \mathrm{~g})$ and adult male New Zealand White rabbits $(3.0 \mathrm{~kg}-3.5 \mathrm{~kg})$ were purchased from the Shandong Lukang animal Center. The animals were housed in groups in stainless steel suspended cages, identified by cards showing the test number and the starting and ending date for the experiment. The temperature of the room was within a range of $20-26^{\circ} \mathrm{C}$. The humidity range of the room was $40-70 \%$. The light cycle was controlled (12 hours light, 12 hours dark). All animal procedures are approved by the Institution Committee of Ethics in Animal Experimentation.

\section{Test Sample and Control Preparation}

Under aseptic condition, silver-based dressings are prepared as test samples $(5 \mathrm{~cm} \times 6 \mathrm{~cm})$, which contain $26.6 \mathrm{mg}$ of silver, and comparable non-silver dressings were prepared accordingly. First, we used an MEM culture containing $10 \%$ FCS to determine the dynamics of silver release at $1 \mathrm{~h}, 6 \mathrm{~h}, 16 \mathrm{~h}, 24 \mathrm{~h}, 48 \mathrm{~h}, 72 \mathrm{~h}$, and $168 \mathrm{~h}$, at $37^{\circ} \mathrm{C}$, with $60 \mathrm{rpm}$ horizontal vibration at a ratio of $3 \mathrm{~cm}^{2} /$ $\mathrm{ml}$, which refers to ISO10992-12:2012. Subsequently, articles of the same size were incubated with an MEM culture containing $10 \%$ FCS, simulated body fluid (SBF), $0.9 \%$ sodium chloride, and 5\% glucose, respectively, under the same conditions. For all preparation processes, the samples should be pre-saturated with $28 \mathrm{~mL}$ of vehicles, based on the previous absorbent capacity test, and then fixed at a ratio of $3 \mathrm{~cm}^{2} / \mathrm{ml}\left(60 \mathrm{~cm}^{2}\right.$ dressings for both sides versus $20 \mathrm{ml}$ vehicles) in the subsequent extraction procedure. Subsequently, each extract was collected and used for silver content determination after 72h incubation.

\section{Silver Determination Using a Graphite Furnace Atomic Absorption Spectrometer (GFAAS)}

$1 \mathrm{ml}$ extracts of dressings were dissolved by $5 \mathrm{ml} \mathrm{HNO}_{3}$ and $2 \mathrm{ml} \mathrm{H}_{2} \mathrm{O}_{2}$. Subsequently, the solutions were diluted with deionized water at 1:10 ratio, and were determined by GFAAS (Thermo Electron Corporation, iCE 3500) under the instruction for use. The sensitivity of the GFAAS is 50ppb. For the silver spiked recovery test, $\mathrm{AgNO}_{3}$ powder was weighed and dissolved by deionized water to a final concentration of $1 \mathrm{mg} / \mathrm{ml}$. The full solution was sterilized by sterile filtration using syringe filters (pore size $\leq 0.22 \mu \mathrm{m}$ ), and analyzed by the GFAAS. For silver-based dressing samples from in vivo tests, we digested the dressing sample $(2 \mathrm{~cm} \times 2 \mathrm{~cm})$ using $7 \mathrm{ml} \mathrm{HNO}_{3}$ and $1 \mathrm{ml}$ hydrofluoric acid before initiating a similar determination process.

\section{Determination of Antimicrobial Efficacy}

C. albicans (ATCC 10231) colonies were diluted and swabbed on conventional agar plates, and were dried for about $6 \mathrm{~min}$. Sterile round pieces of filter paper samples (diameter equal tolcm) were prepared after soaked in $\mathrm{SBF}, 0.9 \%$ sodium chloride, $\mathrm{AgNO}_{3}$ $(1 \mathrm{mg} / \mathrm{ml})$, and $\mathrm{AgNO}_{3}(3 \mu \mathrm{g} / \mathrm{ml})$ extract, respectively, and then set up on the surface of the agar dishes (five per dish). The diameter of the inhibition rings was photographed and measured after $24 \mathrm{~h}$ incubation at $37^{\circ} \mathrm{C}$.

\section{In Vivo Tests}

In vivo tests were performed to demonstrate the silver release overtime. The rats (three animals with one wound each) were anesthetized with $40 \mathrm{mg} / \mathrm{kg}$ of ketamine. Subsequently, one full-thickness incisional wound $(2 \mathrm{~cm} \times 2 \mathrm{~cm})$ was established on the shaved back of each rat using a surgical instrument. Silver based dressings test samples $(2 \mathrm{~cm} \times 2 \mathrm{~cm})$ were set forth and fixed in the wound using self-adhesive elastic bandages. The animals were then moved back to the cage and housed individually under postsurgical animal care procedures. At each scheduled time point, the animals were anesthetized and utilized, then the test samples were taken out, and the residual silver content was analyzed using the AAS method. Accordingly, the released silver is equal to the total indicated silver content in the test samples $(2 \mathrm{~cm} \times 2 \mathrm{~cm})$, minus the residual silver at each scheduled time point. For the irritation test, the procedures specified in ISO 10993-10:2010 Biological evaluation of medical devices -Part 10: Tests for irritation and skin sensitization was fully followed [14]. Healthy young adult albino rabbits are injected intradermally on both sides of the spine with $0.2 \mathrm{ml}$ extracts at each injection site. All erythema and oedema grades at $(24 \pm 2) \mathrm{h},(48 \pm 2) \mathrm{h}$, and $(72 \pm 2) \mathrm{h}$ were added after each injection. The requirements of the test are met if the final test sample score is 1.0 or less.

\section{Statistical Analysis}

The significance of the differences between different groups and controls was analyzed by the Student's t-test using origin 8 software. $\mathrm{P}<0.05$ was regarded as significance. 


\section{Results}

\section{Silver Ion Release Kinetics in the MEM Containing 10\% FCS}

The silver content in the MEM containing 10\% FCS was determined by the GFAAS at each scheduled time point and the AgNO 3 spike recovery test. The results have shown that the silver content in the extract was accumulated overtime and reached its maximum (approximately $1.4 \mathrm{mg}$ ) at $168 \mathrm{~h}$ incubation, which might be close to the total indicated silver content of the sample, as demonstrated in Figure 1.

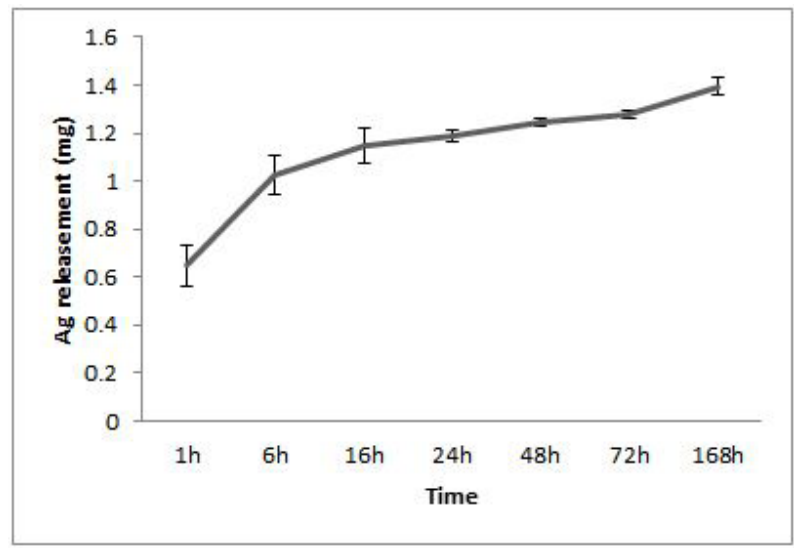

Figure 1: Silver ion release dynamic in the MEM containing 10\% FCS overtime. Results are mean of at least three replicate

\section{Determination of Silver Ion Release in Different Vehicles}

We then determined the silver content in different vehicles after $72 \mathrm{~h}$ incubation under the conditions of $37{ }^{\circ} \mathrm{C}$ with $60 \mathrm{rpm}$ horizontal vibration at a ratio of $3 \mathrm{~cm}^{2} / \mathrm{ml}$. As is shown in Figure 2A, different kinds of vehicles experienced significant differences, which indicate that the highest silver content was in the MEM containing 10\% FCS, while the lowest silver content was in the 5\% glucose. To further investigate the dissimilating capacity of the precipitated silver ions on the surface of the dressings in vitro, we also compared the color changes between the silver based dressing and comparable non-silver based dressings after $72 \mathrm{~h}$ incubation. The results have shown that compared with the control groups, obvious color changes were observed on the surface of the test samples, along with a little bit of silver precipitation and oxidation, except for the $5 \%$ glucose group (Figure 2B). Results from the experiments on irritation of silver release over time (Figure $2 \mathrm{C}$ ) showed that compared with $\mathrm{AgNO}_{3}(1 \mathrm{mg} / \mathrm{ml})$ and $\mathrm{AgNO}_{3}$ $(3 \mu \mathrm{g} / \mathrm{ml})$ control groups, which displayed a serious irritation effect, the score of all the test groups was less than 1.0, indicating no obvious irritation reaction, which were in accordance with the silver release results in vitro. Also, the results of antimicrobial efficacy have shown that, compared with the $\mathrm{AgNO}_{3}(1 \mathrm{mg} / \mathrm{ml})$ control group, no visible inhibition rings were identified by SBF and $0.9 \% \mathrm{NaCl}$ groups (Figure 2D).

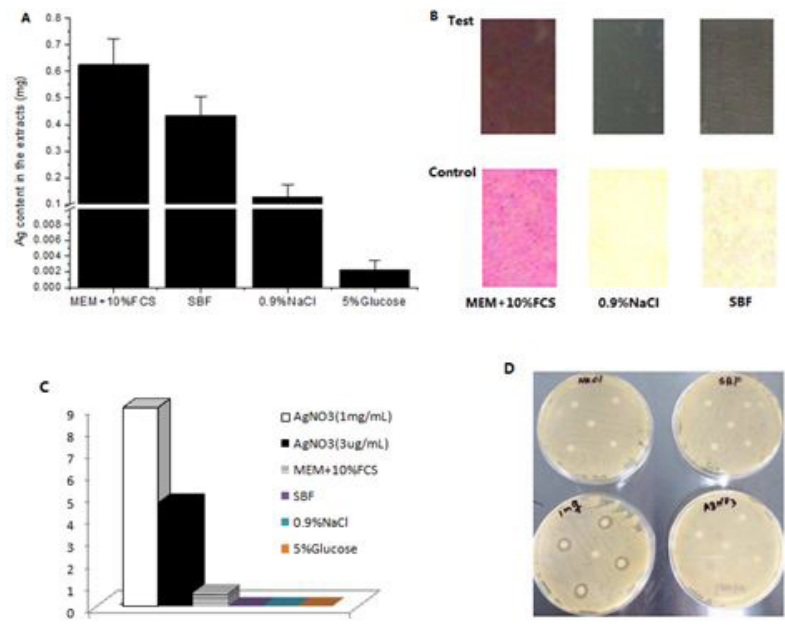

Figure 2: Silver ion release in different kinds of test solutions. (A) Silver contents were analyzed by AAS. Results are mean of at least three replicate. (B) Color changes on the surface of different test and control samples were photographed, respectively. The color of each sample was photographed 30 seconds after the extraction duration. The control samples are from comparator dressings only in lack of silver. (C) The irritation test was performed fully compliant to the protocol specified in ISO 10993-10:2010. Results have shown that the average scores of AgNO ${ }_{3}(1 \mathrm{mg} / \mathrm{ml}$ ) and $\mathrm{AgNO}_{3}(3 \mu \mathrm{g} / \mathrm{ml})$ control groups were 9.0 and 4.7 respectively, while all the scores in different test groups were less than 1.0 . The scores are calculated as follows: After the $(72 \pm 2) \mathrm{h}$ grading, all erythema grades plus oedema grades $(24 \pm 2) \mathrm{h},(48 \pm 2) \mathrm{h}$ and $(72 \pm 2) \mathrm{h}$ are totalled separately for each test sample or blank for each individual animal. To calculate the score of a test sample or blank on each individual animal, divide each of the totals by 15 ( 3 scoring time points $\times 5$ test or blank sample injection sites). Results are mean of at least three rabbits. (D) Inhibition rings were photographed from $\mathrm{AgNO}_{3}(1 \mathrm{mg} / \mathrm{ml})$ and $\mathrm{AgNO}_{3}(3 \mu \mathrm{g} / \mathrm{ml})$ control groups, $\mathrm{SBF}$ and $0.9 \% \mathrm{NaCl}$ test groups, respectively after $24 \mathrm{~h}$ incubation at $37^{\circ} \mathrm{C}$ 


\section{Silver Ion Release In Vivo}

In vivo tests using rat models were performed to determine the silver ion release after $72 \mathrm{~h}$ and $168 \mathrm{~h}$ application, with regard to 3-7 days of a conventional clinical practice. The results have shown that about $81.9 \%$ of silver ions in the testing sample have been released after $72 \mathrm{~h}$ contact, as well as around 98.6 percent of silver ions release after $168 \mathrm{~h}$ exposure, indicating that the silver ions in the dressing had undergone thorough release due to $168 \mathrm{~h}$ continued contact in vivo (Figure 3 ).
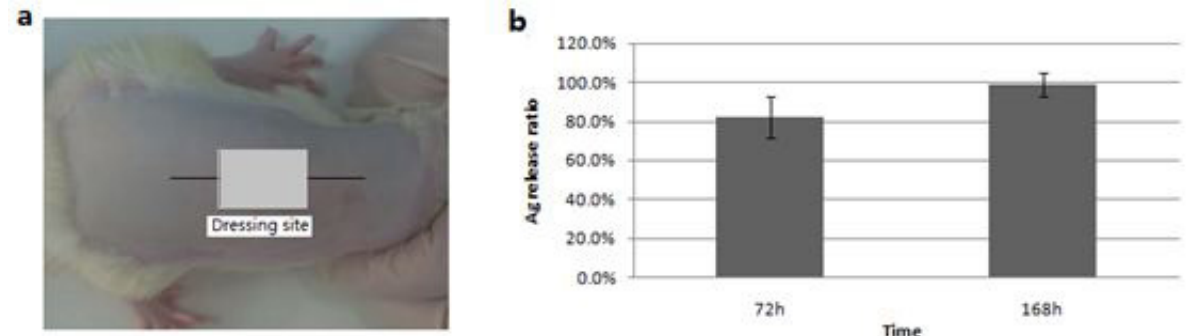

Figure 3: (A) Location of wounds and wound dressings in rat models and (B) Average ratios of silver ion release were calculated after $72 \mathrm{~h}$ and $168 \mathrm{~h}$ application in rat models, respectively. Results are mean of at least three replicate from each group

\section{Discussion}

Over the past decades, increasing numbers of newly designed silver ion based dressings have been developed and approved on the market. However, since the first silver ion based wound dressing was developed and applied in 1987, studies regarding silver content and its release from dressings remain controversial when considering its overall safety and effectiveness [15]. Today, researches on the biocompatibility of silver ion based dressings have become available. During their wide applications for wound healing, cytotoxicity is one of biggest concerns that should be taken into account when deciding safety standards for these dressings [16]. In our previous study, we have demonstrated the correlation between silver ion release and its cytotoxicity [17].

In this study, we used the MEM with $10 \%$ fetal calf serum as the targeted vehicle to illustrate silver ion release kinetics in vitro. As is shown in Figure 1, the total amount of silver ions released into the medium incremented overtime, and reached about 5\% of the total silver content in the dressing sample after $168 \mathrm{~h}$ incubation, which indicated that the MEM with $10 \%$ fetal calf serum could be used as a promising vehicle to simulate the real silver ion release in vitro. To further confirm the suitability of this kind of MEM, we compared it with SBF, $0.9 \%$ sodium chloride, and $5 \%$ glucose. The results showed that the silver ions released into MEM complement medium is a little bit higher than any other different vehicle after $72 \mathrm{~h}$ incubation, which further testifies to the suitability of the MEM complement medium for the determination of silver ion release kinetics in vitro. In addition, our results also showed that the precipitated silver ions on the surface of the dressing samples would be oxidized, and experienced a significant color change in comparison with the non-silver ion based dressings when exposed in the air only for 30 seconds (Figure $2 \mathrm{~B}$ ), which also indicated that the precipitated silver ion on the surface of the dressings could not be dissimilated simultaneously, compared to the in vivo scenarios. In accordance with the irritation test and antimicrobial efficacy that could illustrate the silver ion release from silver based dressings, our results in this study have also shown that the standard intra-dermal reactivity test and antimicrobial efficacy determination could further prove the silver ion release in vitro, regardless of the silver forms in the vehicles (Figure 2C and Figure 2D).

It is well known that silver ion release using the appropriate animal model was the best way to simulate the clinical practice [18]. Thus, from a clinical aspect, we investigated the silver ion release in the rat models (Figure 3), and found that the silver ion release ratio had incremented from $81.9 \%$ to $98.6 \%$ after $72 \mathrm{~h}$ and $168 \mathrm{~h}$ application, respectively, indicating that the silver ion release speed was much faster and higher in vivo than in vitro, which we hypothesized might be related to the mobility of the sample and silver ion elimination in time.

\section{Conclusion}

In conclusion, our studies have confirmed that MEM medium with 10\% fetal calf serum might be used as an appropriate standard vehicle to investigate the silver ion release from dressings in vitro, without considering the interacting model between a silver ion and the loaded dressing matrix [19]. More importantly, a rat model could experimentally simulate the clinical applications in vivo. Further studies will focus on characterizing the combination of a silver ion and dressing matrix to fully identify the impacts for silver ion release and fulfill their safely clinical applications in the future.

\section{Acknowledgement}

The author(s) disclosed receipt of the following financial support for the research, authorship, and/or publication of this article: This work was funded, in part, by Shandong science and technology development plan project (2017GSF218004) and National key research and development project (2016YFC1103205) and (2016YFC1102503). 


\section{References}

1. Burrell RE (2003) A Scientific Perspective on the Use of Topical Silver Preparations. Ostomy Wound Manage 49: 19-24.

2. Maillard JY, Hartemann P (2013) Silver as an antimicrobial: facts and gaps in knowledge. Crit Rev Microbiol 39: 373-83.

3. Parsons D, Bowler PG, Myles V, Jones S (2005) Silver antimicrobial dressings in wound management: a comparison of antibacterial, physical, and chemical characteristics. Wounds A Compend Clin Res Pract 17: 222-32.

4. Rigo C, Roman M, Munivrana I, Vindigni V, Azzena B, et al. (2012) Characterization and evaluation of silver release from four different dressings used in burns care. Burns 38: 1131-42.

5. Schafer B, Tentschert J, Luch A (2011) Nanosilver in Consumer Products and Human Health: More Information Required! Environ Sci Technol 45: 7589-90.

6. Chaby G, Viseux V, Poulain JF, De Cagny B, Denoeux JP, et al. (2005) Topical silver sulfadiazine-induced acute renal failure. Ann Dermatol Venereol 132: 891-3.

7. Atiyeh BS, Costagliola M, Hayek SN, Dibo SA (2007) Effect of silver on burn wound infection control and healing: review of the literature. Burn 33: 139-48.

8. Wright JB, Hansen DL, Burrell RE (1998) The comparative efficacy of two antimicrobial barrier dressings: in-vitro examination of two controlled release of silver dressings. Wounds A Compend Clin Res Pract 10: 179-88.

9. Sofokleous P, Stride E, Edirisinghe M (2013) Preparation, Characterization, and Release of Amoxicillin from Electrospun Fibrous Wound Dressing Patches. Pharm Res 30: 1926-38.

10. Rigo C, Roman M, Munivrana I, Vindigni V, Azzena B, et al. (2012) Characterization and evaluation of silver release from four different dressings used in burns care. Burns 38: 1131-42.

11. Lansdown ABG (2004) A review of the use of silver in wound care: facts and fallacies. British Journal of Nurs 6: 6-19.

12. Percival SL, Bowler PG, Russel D (2005) Bacterial resistance to silver in wound care. J Hosp Infect 60: 1-7.

13. Schierholz JM, Wachol-Drewek Z, Lucas L, Pulverer G (1998) Activity of Silver Ions in different Media. Zentralblatt für Bakteriologie 287: 411-20.

14. ISO 10993-10:2010 Biological evaluation of medical devices-Part 10: Tests for irritation and skin sensitization.

15. Thomas S (2010) Silver Dressings. In: Surgical Dressings and Wound Management. Medetec Publications, Cardiff, South Wales.

16. Poon VK, Burd A (2004) In Vitro Cytotoxicity of Silver: Implication for Clinical Wound Care. Burns 30: 140-7.

17. Liu C, Hou L, Liu L, Qu Z, Wang X, et al. (2016) In Vitro Cytotoxicity Evaluation of Highly Absorbent Foam Dressings Based on Silver Zirconium Phosphate via IC50 Value. J Biomater Nanobiotechnol 7: 37-44.

18. Ignacio C, Barcellos L, Ferreira MD, Moura SA, Soares IA, et al. (2011) In vivo tests of a novel wound dressing based on biomaterials with tissue adhesion controlled through external stimuli. J Mater Sci Mater Med 22: 1357-64.

19. Hemmat Maghsoudi, Siavash Monshizadeh, Mehran Mesgari (2011) A Comparative Study of the Burn Wound Healing Properties of Saline-Soaked Dressing and Silver Sulfadiazine in Rats. Indian J Surg 73: 24-27.

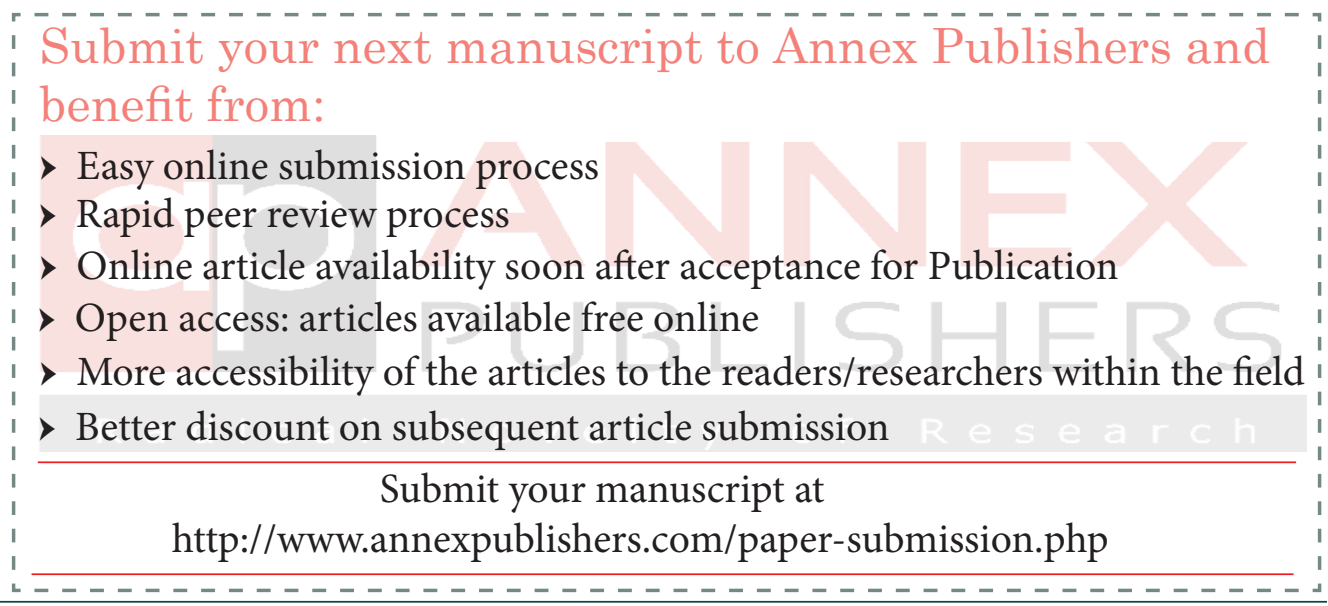

